\title{
Diagnostic Utility of Sago-Like Nodules on Gross Thoracoscopic Appearance in Tuberculous Pleural Effusion and Their Correlation with Final Histo-Microbiologic Findings
}

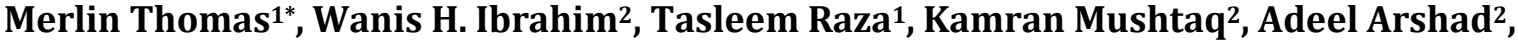 \\ Mushtaq Ahmed ${ }^{2}$, Salma Taha², Shireen Omer ${ }^{2}$, Saber Al Sarafandi' ${ }^{1}$, Omer Rabadi², \\ Hisham A. Abdul-Sattar ${ }^{1}$
}

${ }^{1}$ Department of Chest, Hamad General Hospital, Doha, Qatar

${ }^{2}$ Department of Medicine, Hamad General Hospital, Doha, Qatar

Email: ^mmts1983@gmail.com

How to cite this paper: Thomas, M., Ibrahim, W.H., Raza, T., Mushtaq, K., Arshad, A., Ahmed, M., Taha, S., Omer, S., Al Sarafandi, S., Rabadi, O. and Abdul-Sattar, H.A. (2018) Diagnostic Utility of Sago-Like Nodules on Gross Thoracoscopic Appearance in Tuberculous Pleural Effusion and Their Correlation with Final Histo-Microbiologic Findings. Journal of Tuberculosis Research, 6, 270-280.

https://doi.org/10.4236/jtr.2018.64024

Received: September 23, 2018

Accepted: November 4, 2018

Published: November 7, 2018

Copyright (c) 2018 by authors and Scientific Research Publishing Inc. This work is licensed under the Creative Commons Attribution International License (CC BY 4.0).

http://creativecommons.org/licenses/by/4.0/

(c) (i) Open Access

\begin{abstract}
Background: Distinguishing tuberculous pleural effusion (TPE) from other causes of exudative effusion is often challenging. Delay in treatment initiation can occur while awaiting histo-microbiologic confirmation owing to the paucibacillary nature of the disease. Sago-like nodules are the most common visual finding on gross thoracoscopic appearance. The primary objective was to determine the diagnostic utility of the presence of sago-like nodules on gross thoracoscopic appearance in TPE to help justify early initiation of tuberculosis (TB) treatment based on their finding while awaiting final histo-microbiologic confirmation. Secondary objective was to study the correlation between the presence of sago-like nodules and the final histo-microbiologic findings in pleural biopsy specimens. Methods: This was a retrospective-descriptive study of all patients with exudative pleural effusion who underwent diagnostic medical thoracoscopy (MT) at Hamad General Hospital during an eight-year period (from January, 2008 to December, 2015). Results: The presence of sago-like nodules on gross thoracoscopic appearance of the pleural surface had a sensitivity of $58 \%$, a specificity of $89 \%$ and a positive predictive value of $97 \%$ for TPE with a diagnostic accuracy of $62 \%$. There is significant association between the presence of sago-like nodules and demonstration of granulomatous inflammation in pleural biopsy specimens $(\mathrm{P}=0.000)$. There is no association between sago-like nodules and positive TB smear and culture in biopsy specimens. Conclusion: The presence of sago-like nodules on gross
\end{abstract}


thoracoscopic appearance has a high specificity and positive predictive value for TPE and significantly correlates with the presence of granulomatous inflammation. Patients from TB prevalent areas with exudative pleural effusion and sago-like nodules on gross thoracoscopic appearance may be commenced on TB chemotherapy while awaiting final histologic confirmation.

\section{Keywords}

Tuberculous Pleural Effusions, Medical Thoracoscopy, Sago-Like Nodules

\section{Introduction}

TB poses a major global public health problem for both developing and developed countries. It is the ninth leading cause of death worldwide and the leading cause from a single infectious agent. In 2016, there were an estimated 1.3 million TB deaths among Human Immunodeficiency Virus (HIV)-negative people and an additional 374,000 deaths among HIV-positive with an estimated 10.4 million people fell ill with TB in the same year [1]. Prompt and accurate diagnosis of TB, followed by provision of treatment in line with international standards does not only prevent deaths and limit ill-health among people who develop the disease but also prevents further transmission of infection to others [1]. TPE is the second most common form of extrapulmonary $\mathrm{TB}$ worldwide and the most common cause of exudative pleural effusion in endemic TB areas [2]. TPE typically presents as an acute or subacute illness with fever and unilateral exudative pleural effusion with predominant lymphocyte count in the pleural fluid [2] [3] [4]. Nevertheless, such clinical presentation and initial fluid analysis results can be seen in other diseases such as malignancies, sarcoidosis and some rheumatologic disorders [5]. The gold-standard for the diagnosis of TPE remains the detection of Mycobacterium tuberculosis (MTB) in pleural fluid or pleural biopsy specimens, either by microscopy and/or culture, or the histological demonstration of caseating granulomas in the pleura along with acid fast bacilli (AFB) [4]. Nevertheless, major challenges encountered in confirming the diagnosis of TPE are the failure to detect AFB on microscopic examination in more than $90 \%$ of cases and the long time for AFB to grow on culture (more than 8 weeks) [4]. This can lead to a significant delay in diagnosis and treatment initiation. As a result, the demonstration of AFB has been dropped as an absolute requirement for making the diagnosis of TPE and the presence of caseating granulomas in high burden settings is considered adequate to make the diagnosis [4] [6]. Considering the above challenges in diagnosing TPE, there has been an interest in utilizing the gross thoracoscopic visual appearance of the pleural cavity in making quick diagnosis of TPE in order to initiate the treatment while awaiting final microscopic or histologic confirmation. However, the currently available evidence supporting this approach is limited [7]. We have shown in a previous study from this country that sago-like nodules to be among the most common 
findings on gross thoracoscopic appearance of TPE cases (seen in 51\% of cases) [8]. Similar results have also been reported by other investigators [7]. To the best of our knowledge the diagnostic utility of the presence of sago-like nodule on gross thoracoscopic appearance in making TPE diagnosis is seldom reported in literature. The primary objective of this study was to determine the diagnostic utility of the presence of sago-like nodules on gross thoracoscopic appearance in TPE in order to justify early initiation of TB treatment based on finding of these nodules while awaiting final histo-microbiologic confirmation. Secondary objective was to study the correlation between the presence of sago-like nodules and the final histo-microbiologic findings in pleural biopsy specimens.

\section{Materials and Methods}

This was a retrospective-descriptive study of all patients with exudative pleural effusion who underwent diagnostic MT at Hamad General Hospital (The only tertiary government hospital performing MT in the State of Qatar) during an eight-year period (from January, 2008 to December, 2015).

\subsection{Definition of TPE}

In this study, we defined TPE in a patient with clinical presentation suggestive of TB and exudative pleural effusion by the presence of one or more of the following criteria: 1) positive AFB smear or culture in the pleural fluid or pleural biopsy, 2) presence of caseating granulomas in pleural biopsy; 3) presence of non-caseating epithelioid granulomas in pleural biopsy, in absence of other causes of non-caseating granuloma and favorable response to anti-tuberculous medications.

\subsection{Definition of Sago-Like Nodules}

Sago-like nodules (also called sago grain-like nodules) are fairly small less than 5 $\mathrm{mm}$ solid or caseous seeding of the pleural surface resembling sago grains obtained from palm stems (Figure 1).

\subsection{MT Procedure}

MT at Hamad General Hospital is conducted in the bronchoscopy suite under conscious sedation and local anesthesia using a rigid thoracoscope. The position of the patient varies according to site of effusion, but an anterolateral thoracic approach in the $5^{\text {th }}$ or $6^{\text {th }}$ intercostal space is employed in the majority of patients. Following a $1-2 \mathrm{~cm}$ incision, a blunt dissection is done with an artery forceps. A trocar with inner diameter of $8 \mathrm{~mm}$ is then inserted through a cannula, followed by insertion of the thoracoscope. After achieving clear visualization, the pleural cavity is thoroughly inspected and usually $4-5$ biopsies are taken from the abnormal areas on parietal pleura by a lateral "lift and peel" technique. Multiple biopsies are taken if no gross abnormalities are noted. All samples are submitted for histopathology and mycobacterial culture. After thoracoscopy, a 24 French chest tube connected to an underwater seal is routinely inserted for 


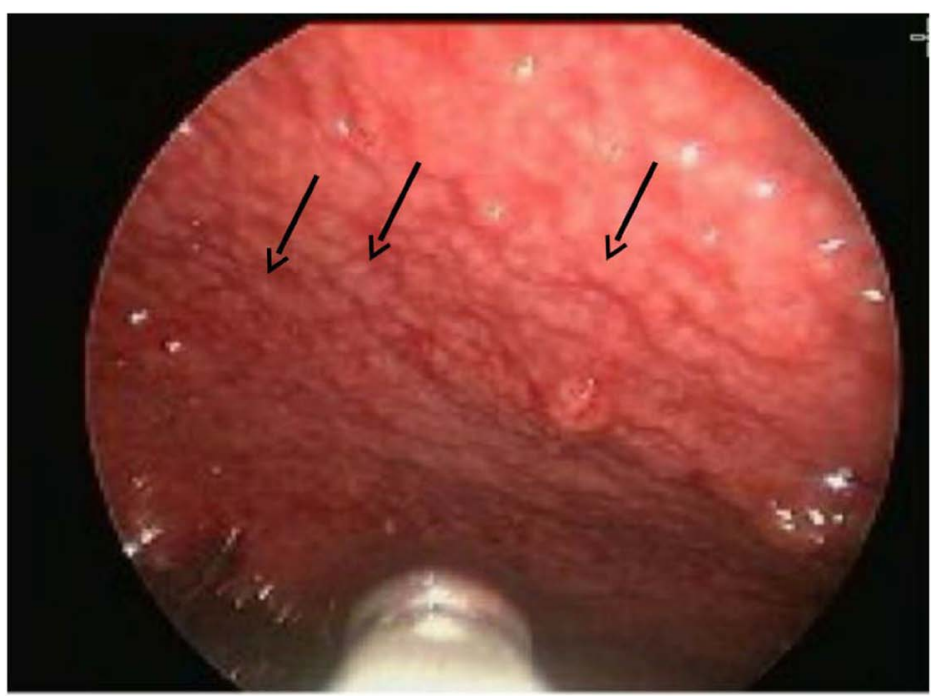

Figure 1. Thoracoscopic image of sago-like nodules on parietal pleura.

drainage of iatrogenic pneumothorax and the tube is removed once pneumothorax has resolved.

\subsection{Data Collection}

Medical records (both paper and electronic) of all patients with exudative pleural effusion who underwent MT during the study period were analyzed. Demographic data of subjects, pleural fluid characteristics, pathological and microbiological details were recorded in a structured data collection sheet. Details of the MT procedure including gross visual thoracoscopic findings and complications were obtained from the electronic as well as the paper health records and recorded in the structured data collection sheet.

\subsection{Statistical Analysis}

Categorical and continuous values were expressed as frequency (percentage) and mean \pm SD or median. Descriptive statistics were used to summarize demographic and all other clinical characteristics of the study subjects. Associations between two or more qualitative variables were assessed using the chi-square test or Fisher Exact test as appropriate. Relationship between two quantitative variables was examined using Pearson's correlation coefficients. All $\mathrm{P}$ values presented are two-tailed, and $\mathrm{P}$ values $<0.05$ were considered statistically significant. All Statistical analyses were done using statistical packages SPSS 22.0 (SPSS Inc. Chicago, IL). The accuracy of gross thoracoscopic findings was expressed as sensitivity, specificity, positive and negative predictive values, and diagnostic accuracy.

\subsection{Ethical Approval}

The research protocol (RP\#14422/14) was approved by the Medical Research Center at Hamad Medical Corporation. 


\section{Results}

Among the 407 subjects with exudative pleural effusion who underwent MT during the study period, 344 were diagnosed with TPE based on the criteria mentioned above. Characteristics of subjects with TPE are shown in Table 1. Sago-like nodules (Figure 1) were observed in 58\% (198/344) of TPE cases and in $11 \%(7 / 63)$ of non-TB cases of exudative pleural effusion (Table 2). The presence of sago-like nodules on gross thoracoscopic appearance of the pleural surface had a sensitivity of $58 \%$, a specificity of $89 \%$ and a positive predictive value of $97 \%$ for TPE with a diagnostic accuracy of $62 \%$ (Table 3 ). Among the 7 patients with non-tuberculous pleural effusion who had sago-like nodules, 4 had metastatic adenocarcinoma, 1 had lymphoma, 1 had para-pneumonic effusion and 1 had lupus pleuritis. The histopathologic examination of pleural biopsy revealed necrotizing granuloma in $76 \%$ (Figure 2), and non-necrotizing granuloma in $12 \%$ of cases (Table 4). Positive AFB smear was observed in $26 \%$ of the pleural biopsy specimens and positive MTB culture in $53 \%$ (Table 5). The presence of sago-like nodules on gross visual appearance during MT is significantly associated with the finding of granulomatous inflammation in pleural biopsy specimens $(\mathrm{P}=0.000)$ (Table 5$)$. However there was no association between sago-like nodules and positive AFB smear and TB culture in biopsy specimens.

Table 1. Characteristics of subjects with TPE.

\begin{tabular}{cc}
\hline Demographics variables & N (\%) (Total 344) \\
Age & $30.8 \pm 9.5$ years \\
Mean age & $14(4.1)$ \\
$<20$ years & $310(89.1)$ \\
20 - 50 years & $16(4.7)$ \\
$\mathbf{5 0}-\mathbf{6 4}$ years & $4(1.2)$ \\
$>65$ years & \\
Gender & $301(87.5)$ \\
Male & $43(12.5)$ \\
Female & \\
Ethnicity & $267(77.6)$ \\
Indian subcontinent & $24(7.0)$ \\
Philippines & $11(3.2)$ \\
Middle east & $33(9.6)$ \\
African & $9(2.6)$ \\
Other & $180(64.3)$ \\
Occupation & $64(22.9)$ \\
Unskilled & $24(8.6)$ \\
Semiskilled & \\
Skilled & \\
& \\
\hline & \\
\hline & \\
\hline & \\
\hline & \\
\hline
\end{tabular}




\section{Continued}

Others

$12(4.3)$

Risk factors \& Comorbidities

Smoking

Alcohol

Diabetes Mellitus

$34(10)$

$9(2.6)$

Hypertension

Coronary Artery disease

Average length of hospital stay (days)

Table 2. Gross thoracoscopic findings in TPE.

\begin{tabular}{cc}
\hline Finding & N (\%) \\
\hline Normal & $11(3.2 \%)$ \\
Hyperemic pleura only & $11(3.2 \%)$ \\
Hyperemia with adhesions & $101(29.4 \%)$ \\
Sago-like nodules with hyperemia & $198(57.5 \%)$ \\
Hyperemia with non-Sago like nodules/plaque like lesions & $8(2.3 \%)$ \\
Missing info & $15(4.4)$
\end{tabular}

Table 3. Diagnostic value of sago-like nodules for TPE.

\begin{tabular}{cc}
\hline Sago like nodules in TPE & \\
\hline Sensitivity & $58 \%$ \\
Specificity & $89 \%$ \\
Positive Predictive value & $97 \%$ \\
Negative predictive Value & $28 \%$ \\
Positive Likelihood Ratio (LR+) & 5.2 \\
Negative Likelihood Ratio (LR-) & 4.8 \\
Diagnostic accuracy & $62 \%$ \\
\hline
\end{tabular}

Table 4. Histo-Microbiologic findings in pleural biopsy specimens in TPE.

\begin{tabular}{cc}
\hline Histopathology & N (\%) \\
\hline Necrotizing Granuloma & $262(76.2)$ \\
Non Necrotizing Granuloma & $41(12)$ \\
Non Specific Inflammation & $31(9)$ \\
Normal & $4(1.1)$ \\
Inadequate & $6(1.7)$ \\
Microbiology & $\mathbf{N}(\%)$ \\
Positive AFB stain of pleural Biopsy $(\mathrm{N}=\mathbf{3 3 6})$ & $88(25.5)$ \\
Positive Tb culture of pleural Biopsy $(\mathrm{N}=\mathbf{2 0 9})$ & $114(53.1)$
\end{tabular}


Table 5. Association between the presence of Sago-like nodules on gross thoracoscopic appearance and presence of granuloma, positive AFB smear and positive TB culture.

\begin{tabular}{ccccc}
\hline Outcome & \multicolumn{3}{c}{$\begin{array}{c}\text { Sago-like nodules present Sago-like nodules absent } \\
\text { on thoracoscopy }\end{array}$} & P value \\
\hline $\begin{array}{c}\text { Granuloma in } \\
\text { pleural biopsy } \\
\text { present }\end{array}$ & $\begin{array}{c}\text { Granuloma } \\
\text { absent }\end{array}$ & 22 & 100 & $\mathbf{0 . 0 0 0}$ \\
& $\begin{array}{c}\text { AFB smear } \\
\text { positive }\end{array}$ & 51 & 57 & $\mathbf{0 . 4 2 4}$ \\
$\begin{array}{c}\text { AFB smear in } \\
\text { pleural biopsy } \\
\text { AFB smear } \\
\text { negative }\end{array}$ & 150 & 33 & \\
$\begin{array}{c}\text { TB culture } \\
\text { positive }\end{array}$ & 64 & 119 & $\mathbf{0 . 1 0 2}$ \\
TB culture in & $\begin{array}{c}\text { TB culture } \\
\text { negative }\end{array}$ & 56 & 61 & \\
\hline
\end{tabular}

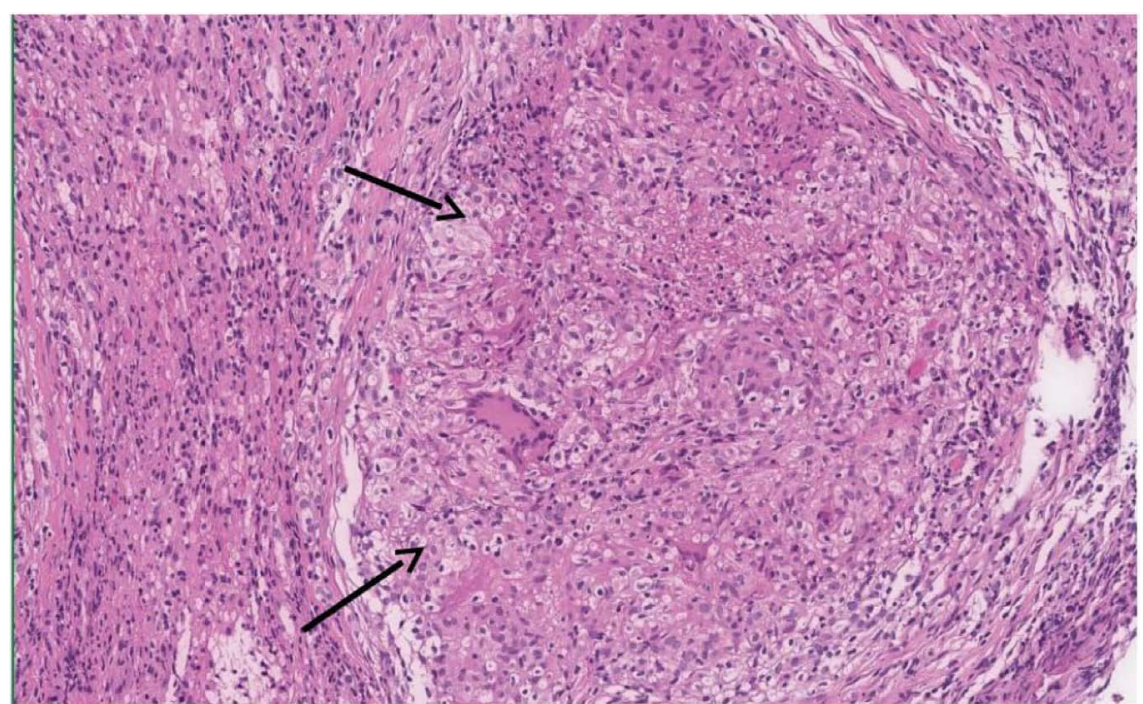

Figure 2. Necrotizing granuloma in tuberculous pleuritis showing a peripheral rim of epithelioid histiocytic surrounding the central necrotic region ( $\mathrm{H} \& \mathrm{E}, 400 \times)$.

\section{Discussion}

Pinpointing the cause of exudative pleural effusion in a patient poses a diagnostic challenge, particularly in distinguishing TPE from other causes of exudative effusion. Because the conventional investigations done on pleural fluid are not always helpful, more invasive procedures are usually needed to confirm the diagnosis and exclude other diseases that cause exudative effusion [9]. MT has gained widespread acceptance in the diagnosis of exudative pleural effusion as it allows direct visualization of the pleura and biopsy from abnormal sites. In many institutions where facilities for MT are available, it replaces second-attempt thoracentesis and closed needle biopsy for patients with exudative effusions of unclear etiology [10]. The visual features are the first and most direct findings on 
MT. As histological verification usually takes 5 - 7 days in many hospitals [7], visual features on MT can afford physicians the early impression about the diagnosis, help them pursue the management of patients and minimize delay in treatment and possibly length of hospital stay [7]. Studies conducted on peritoneal TB proved that visual diagnosis on laproscopic examination was very accurate in making the diagnosis of this type of TB. Such studies recommended the initiation of TB chemotherapy on the basis of visual laparoscopic appearances alone [11] [12]. Nevertheless, the evidence on utility of thoracoscopic visual appearance in the diagnosis of TPE is limited by the scarcity of studies. In the current study, sago-like nodules along with hyperermia was the most common gross thoracoscopic finding in TPE. Similar results have also been reported by other investigators. In a study of 46 patients with TPE who underwent MT, Casalini et al. reported that presence of small nodules on the parietal pleura as the most frequent endoscopic picture at thoracoscopy in 56.5\% of cases [13]. Similarly, Wang et al. reported small pleural nodules as the most common gross thoracoscopic finding in $69.4 \%$ of cases [14]. In this study also, we demonstrated the high specificity and positive predictive value of sago-like nodules in diagnosing TPE. In an agreement with this finding, Kong et al. in a retrospective study from China evaluated 91 patients who underwent MT for suspected TPE and reported a sensitivity and specificity of the presence of diffuse miliary nodules on pleural surface as $64.47 \%$ and $86.67 \%$ respectively with a positive predictive value of 96.08\% [7]. An interesting end point in the current study is investigating the correlation between the presence of sago-like nodules and the final histologic findings on pleural biopsy. The presence of sago-like nodules was significantly associated with the demonstration of granuloma formation in pleural biopsy but not with positive AFB smear or culture. To the best of our knowledge this correlation is seldom reported in previous studies. Granulomas are the hallmark of MTB infection and thus sit at the center of TB immunopathogenesis. They are compact, organized aggregates of immune cells consisting of blood-derived infected and uninfected macrophages, foamy macrophages, epithelioid cells (uniquely differentiated macrophages), and multinucleated giant cells (Langerhans cells) surrounded by a ring of lymphocytes. The granuloma's main function is to localize and contain MTB while concentrating the immune response to a limited area [15] (Figure 2). In the current study granuloma formation in pleural biopsy was observed in $88 \%$ of cases while positive AFB smear in $25 \%$ and positive AFB culture in 53\%. This finding confirms the previous recommendation that demonstration of MTB bacilli in biopsy specimens is not an absolute requirement for making TPE diagnosis and the presence of caseating granulomatous inflammation in high burden sittings is considered adequate [4] [6] [16]. In the State of Qatar, TB is a common health problem with an incidence of $40 / 100,000$ populations per year and is the most common etiology of exudative pleural effusions accounting for $84.5 \%$ of cases [8]. About $97 \%$ of TB patients are expatriates (mostly from Asian countries with high TB prevalence) [17]. Due to the high 
burden of TB, most patients with exudative pleural effusions are admitted to the hospital for isolation until sputum results are proved negative for AFB and MT is usually performed in hospital. These factors could have attributed to the high average length of stay associated with MT procedure in the current study. Following MT procedure, waiting for histologic confirmation (can take up to one week) may further result in substantial delay in initiating TB chemotherapy. The high specificity and high positive predictive value of sago-like nodules may thus justify the initiation of TB chemotherapy while awaiting final histological conformation. Besides being among the first studies reporting the diagnostic utility of sago-like nodules in making TPE diagnosis and elaborating on the correlation with final histologic findings, a point of strength in the current study is the relatively large number of recruited patients compared to previous studies investigating the role of MT in diagnosing pleural effusion. Furthermore, the current study confirmed the results of previous studies regarding the prevalence and diagnostic utility of sago-like nodule in TPE. Nevertheless, besides the retrospective nature, important limitation of this study is the lack of study of the impact of operator (performing physician) experience on the visual thoracoscopic findings in TPE. Furthermore, because estimation of adenosine deaminase (ADA) level in pleural fluid is not performed in our institution, we could not study the correlation between ADA level and the presence of sago-like nodules.

\section{Conclusion}

In conclusion, the presence of sago-like nodules on gross thoracoscopic appearance has a high specificity and positive predictive value for TPE and significantly correlates with the presence of granulomatous inflammation. Patients from TB prevalent areas with exudative pleural effusion and sago-like nodules on gross thoracoscopic appearance may be commenced on TB chemotherapy while awaiting final histologic confirmation.

\section{Acknowledgements}

We would like to thank Dr. Prem Chandra, Biostatistician, Medical Research Centre, Hamad Medical Corporation, Doha, Qatar for his assistance with the statistical analysis in this research article.

\section{Conflicts of Interest}

This manuscript is an original one. It has not been published or considered for publication elsewhere. None of the authors has financial or otherwise any conflict of interest from publishing this manuscript. The manuscript has been seen and agreed upon by all authors.

\section{References}

[1] (2017) Global Tuberculosis Report 2017. World Health Organization, Geneva. http://apps.who.int/iris/bitstream/handle/10665/259366/9789241565516-eng.pdf?se 
quence $=1$

[2] Zhai, K., Lu, Y. and Shi, H.-Z. (2016) Tuberculous Pleural Effusion. Journal of Thoracic Disease, 8, E486-E494. https://doi.org/10.21037/jtd.2016.05.87

[3] Ibrahim, W.H., Ghadban, W., Khinji, A., Yasin, R., Soub, H., Al-Khal, A.L. and Bener, A. (2005) Does Pleural Tuberculosis Disease Pattern Differ among Developed and Developing Countries? Respiratory Medicine, 99, 1038-1045. https://doi.org/10.1016/j.rmed.2004.12.012

[4] Vorster, M.J., Allwood, B.W., Diacon, A.H. and Koegelenberg, C.F. (2015) Tuberculous Pleural Effusions: Advances and Controversies. Journal of Thoracic Disease, 7, 981-991.

[5] Hooper, C., Gary Lee, Y.C. and Maskell, N., On Behalf of the BTS Pleural Guideline Group (2010) Investigation of a Unilateral Pleural Effusion in Adults: British Thoracic Society Pleural Disease Guideline 2010. Thorax, 65, ii4eii17.

[6] Diacon, A.H., Van de Wal, B.W., Wyser, C., Smedema, J.P., Bezuidenhout, J., Bolliger, C.T., et al. (2003) Diagnostic Tools in Tuberculous Pleurisy: A Direct Comparative Study. European Respiratory Journal, 22, 589-591. https://doi.org/10.1183/09031936.03.00017103a

[7] Kong, X.L., Zeng, H.H., Chen, Y., Liu, T.T., Shi, Z.H., Zheng, D.Y., et al. (2014) The Visual Diagnosis of Tuberculous Pleuritis under Medical Thoracoscopy: A Retrospective Series of 91 Cases. European Review for Medical and Pharmacological Sciences, 18, 1487-1495.

[8] Thomas, M., Ibrahim, W.H., Raza, T., Mushtaq, K., Arshad, A., Ahmed, M., et al. (2017) Medical Thoracoscopy for Exudative Pleural Effusion: An Eight-Year Experience from a Country with a Young Population. BMC Pulmonary Medicine, 17, 151.

[9] Liu, J.Y., Xiong, L., Zhang, M., Shuai, S.Y., Wei, X.S., Ye, L.L., et al. (2017) Medical Thoracoscopy in China-The Present Status and the Future. Journal of Thoracic Disease, 9, 406-413. https://doi.org/10.21037/jtd.2017.02.39

[10] Lee, P. and Colt, H.G. (2013) Pleuroscopy in 2013. Clinics in Chest Medicine, 34, 81-91. https://doi.org/10.1016/j.ccm.2012.11.003

[11] Hong, K.D., Lee, S.I. and Moon, H.Y. (2011) Comparison between Laparoscopy and Noninvasive Tests for the Diagnosis of Tuberculous Peritonitis. World Journal of Surgery, 35, 2369-2375. https://doi.org/10.1007/s00268-011-1224-2

[12] Bhargava, D.K., Shriniwas, C.P., Nijhawan, S., Dasarathy, S. and Kushwaha, A.K. (1992) Peritoneal Tuberculosis: Laparoscopic Patterns and Its Diagnostic Accuracy. The American Journal of Gastroenterology, 87, 109-112.

[13] Casalini, A.G., Mori, P.A., Majori, M., Anghinolfi, M., Silini, E.M., Gnetti, L., et al. (2018) Pleural Tuberculosis: Medical Thoracoscopy Greatly Increases the Diagnostic Accuracy. ERJ Open Research, 4, pii: 00046-2017.

[14] Wang, Z., Xu, L.L., Wu, Y.B., Wang, X.J., Yang, Y., Zhang, J., et al. (2015) Diagnostic Value and Safety of Medical Thoracoscopy in Tuberculous Pleural Effusion. Respiratory Medicine, 109, 1188-1192. https://doi.org/10.1016/j.rmed.2015.06.008

[15] Guirado, E. and Schlesinger, L.S. (2013) Modeling the Mycobacterium tuberculosis Granuloma-The Critical Battlefield in Host Immunity and Disease. Frontiers in Immunology, 4, 98. https://doi.org/10.3389/fimmu.2013.00098

[16] Valdés, L., Alvarez, D., San José, E., Penela, P., Valle, J.M., García-Pazos, J.M., et al. (1998) Tuberculous Pleurisy: A Study of 254 Patients. JAMA Internal Medicine, $158,2017-2021$. 
[17] Khattab, M.A., Khan, F.Y., Al Maslamani, M., Al-Khal, A.L., El Gendy, A., Al Soub, H., et al. (2015) Pulmonary and Extra Pulmonary Tuberculosis in Qatar: A First Retrospective Population-Based Study. Advances in Infectious Diseases, 5, 148-153. https://doi.org/10.4236/aid.2015.54018 